\title{
Dúvidas e medos de adolescentes acerca da sexualidade e a importância da educação sexual na escola
}

\author{
Doubts and fears of adolescents about sexuality and the importance of sex education in \\ school
}

Dudas y temores de los adolescentes sobre la sexualidad y la importancia de la educación sexual en la escuela

Luciana Uchôa Barbosa ${ }^{1 *}$, Juliana de Castro Nunes Pereira ${ }^{1}$, Angélica de Godoy Torres Lima ${ }^{1}$, Suzana Santos da Costa ${ }^{2}$, Raylane da Silva Machado ${ }^{3,4}$, Amanda Haissa Barros Henriques ${ }^{5}$, Vanderlei Folmer ${ }^{6}$.

\section{RESUMO}

Objetivo: Identificar dúvidas e medos sobre sexualidade e analisar a percepção dos adolescentes acerca do tema. Métodos: Pesquisa descritiva, qualitativa com adolescentes do $6^{\circ}$ ao $9^{\circ}$ ano do ensino fundamental, por meio de questionário autoaplicável com perguntas abertas sobre dúvidas e medos acerca do tema. Resultados: Participaram 38 adolescentes, a maioria do sexo feminino, com faixa etária média de 13 anos. As principais dúvidas sobre sexualidade voltaram-se para os termos "dúvidas", "doença" e "AIDS". Quanto aos medos dos adolescentes em relação a sua sexualidade, apontou-se os termos "doença", "engravidar" e "medo" com grande frequência nas respostas. Foi possível observar que questões afetivas e sociais permeiam o universo da sexualidade vivenciada por estes. Conclusão: Observou-se que o medo em contrair IST e da gravidez precoce, bem como as dúvidas quanto as questões que envolvem a sexualidade, estão muito presentes entre os adolescentes. Compreende-se a necessidade de prática mais efetiva da educação sexual no contexto escolar com a participação dos adultos de referência, de maneira transversal, para que o adolescente possa adotar comportamentos seguros e saudáveis, fortalecendo o empoderamento e impactando diretamente na redução de indicadores de IST's e gravidez precoce, que são situações que interferem na qualidade de vida dos adolescentes.

Palavras-chave: Adolescente, Educação, Saúde, Sexualidade.

\footnotetext{
ABSTRACT

Objective: Identify doubts and fears about sexuality and to analyze the adolescents perception about the theme. Methods: Descriptive, qualitative research with adolescents from the 6th to the 9th grade of elementary school, through a self-administered questionnaire with open questions about doubts and fears about the theme. Results: 38 adolescents participated, most of them female, with an average age of 13 years. The main doubts about sexuality turned to the terms "doubts", "disease" and "AIDS". As for the adolescents fears regarding their sexuality, the terms "disease", "getting pregnant" and "fear" were frequently mentioned in the responses. It was possible to observe that affective and social issues permeate the

${ }^{1}$ Instituto Federal de Educação, Ciência e Tecnologia de Pernambuco (IFPE), Belo Jardim - PE.

*E-mail: luciana.uchoa@belojardim.ifpe.edu.br

2 Instituto Federal de Educação, Ciência e Tecnologia de Pernambuco (IFPE), Abreu e Lima - PE.

3 Universidade Federal do Piauí, Teresina - PI.

${ }^{4}$ Colégio Técnico de Bom Jesus, Bom Jesus - PI.

${ }^{5}$ Instituto Federal de Educação, Ciência e Tecnologia da Paraíba (IFPB), campus Mangabeira, João Pessoa $-\mathrm{PB}$

${ }_{6}$ Universidade Federal do Pampa - UNIPAMPA, Uruguaiana - RS.
} 
universe of sexuality experienced by them. Conclusion: It was observed that the fear of contracting STIs and early pregnancy, as well as doubts about the issues surrounding sexuality, are very present among adolescents. The need for more effective practice of sex education in the school context is understood with the participation of reference adults, in a transversal way, so that adolescents can adopt safe and healthy behaviors, strengthening empowerment and directly impacting the reduction of STIs indicators and early pregnancy, which are situations that interfere with the quality of life of adolescents.

Key words: Adolescent, Education, Health, Sexuality.

\section{RESUMEN}

Objetivo: Identificar dudas y temores sobre la sexualidad y analizar la percepción de los adolescentes sobre el tema. Métodos: Investigación descriptiva y cualitativa con adolescentes de $6^{\circ}$ a $9^{\circ}$ grado de primaria, a través de un cuestionario autoadministrado con preguntas abiertas sobre dudas y temores sobre el tema. Resultados: Participaron 38 adolescentes, la mayoría mujeres, con una edad promedio de 13 años. Las principales dudas sobre la sexualidad se centraron en los términos "dudas", "enfermedad" y "SIDA". En cuanto a los principales temores de las adolescentes con respecto a su sexualidad, los términos "enfermedad", "quedar embarazada" y "miedo" se mencionaron con frecuencia en las respuestas. Fue posible observar que los problemas afectivos y sociales impregnan el universo de la sexualidad que experimentan. Conclusión: Se observó que el miedo a contraer ITS y el embarazo temprano, así como las dudas sobre los problemas relacionados con la sexualidad, están muy presentes entre los adolescentes. La necesidad de una práctica más efectiva de educación sexual en el contexto escolar se entiende con la participación de adultos de referencia, de manera transversal, para que el adolescente pueda adoptar comportamientos seguros y saludables, fortaleciendo el empoderamiento e impactando directamente la reducción de los indicadores de ITS y embarazo precoz, que son situaciones que interfieren con la calidad de vida de los adolescentes.

Palabras clave: Adolescente, Educación, Salud, Sexualidad.

\section{INTRODUÇÃO}

A adolescência caracteriza-se como uma fase peculiar do ciclo vital humano, assinalada pela passagem da infância para a idade adulta. $O$ indivíduo vivencia mudanças físicas, cognitivas e emocionais com o surgimento dos caracteres sexuais secundários, o advento da menarca no sexo feminino selando o início do ciclo reprodutivo e a descoberta de novas relações intrapessoais, interpessoais e com o meio; sendo um período de amadurecimento da identidade pessoal e sexual do indivíduo (CAMPOS HM, 2011; FAIAL LCM, et al., 2016; LINS LS, et al., 2017). Esse é um momento em que o jovem busca novas experiências, testa seus limites e a afirmação de sua identidade, através do questionamento de seus valores e sonhos. $\mathrm{Na}$ procura pela sua autonomia torna-se importante o afastar-se da família para estar perto de seus amigos, com quem compartilha suas descobertas e medos (TORQUATO BGS, et al., 2017).

A experimentação da sexualidade geralmente ocorre nessa fase da vida, sendo definida em seu sentido mais amplo como a energia contida no ser humano envolvendo práticas e desejos interligados a diferentes formas de sentir prazer e se satisfazer, sendo algo que é independente do sexo e da idade (LINS LS, et al., 2017). A sexualidade é um componente representativo na construção da identidade dos adolescentes, que se manifesta por diversas facetas, tais como a imagem corporal, a descoberta do outro como objeto de amor e desejo e da descoberta de si das relações com familiares e grupos (TORQUATO BGS, et al., 2017).

A educação sexual é essencial para que o adolescente perceba que dispõe de apoio dos adultos de referência (família, professores ou profissionais de saúde) para trocar informações corretas sobre o assunto e possam ter uma sexualidade saudável e livre de dúvidas e medos. $\mathrm{O}$ adolescente ao perceber que sua vida sexual está se iniciando tem a necessidade de desenvolver segurança para uma sexualidade saudável, entretanto, existem fatores que interferem na efetivação de estratégias que garantam o desenvolvimento desta (QUEIROZ VR e ALMEIDA JM, 2017).

A família por se relacionar de forma mais íntima com o adolescente, exerce uma função essencial na construção da sexualidade através da passagem de valores e práticas a partir do diálogo, ajudando a 
consolidar a formação do indivíduo e minimizando as atitudes de risco (LINS LS, et al., 2017). Entretanto, uma parcela significativa dos pais revela apresentar dificuldade em educar seus filhos quanto ao tema sexualidade, devido a insegurança para se comunicarem quanto ao assunto por ser considerado delicado e difícil de ser abordado, ou pela desatenção e aparente falta de interesse dos filhos (TORQUATO BGS, et al., 2017).

Em muitos casos, observa-se que essa responsabilidade é transferida exclusivamente para os educadores, que discutem o assunto mesmo estando despreparados, ao abordarem apenas o enfoque biológico, desprezando a parte psicossocial e cultural, demonstrando que a temática ainda não é explorada de maneira satisfatória no contexto escolar e encontra-se cercada de mistérios e tabus (QUEIROZ VR e ALMEIDA JM, 2017).

Compreende-se que há um prejuízo na dinâmica do acolhimento, aumentando a distância entre as propostas elaboradas e as carências da adolescência, visto que este público manifesta interesse em discutir mais do que a prevenção das Infecções Sexualmente Transmissíveis (IST's) e gravidez, mas também demonstraram interesse e dúvidas sobre a escolha do parceiro e o momento ideal para iniciar as relações sexuais ou como saber a hora certa de viver essa experiência, ou seja, discutir o tema da sexualidade na perspectiva das relações afetivas e amorosas. Dessa forma, é preciso rever práticas de saúde e educação direcionadas a essa parcela vulnerável da sociedade (FAIAL LCM, et al., 2016; QUEIROZ VR e ALMEIDA JM, 2017; MACEDO EOS e CONCEIÇÃO MIG, 2015).

O espaço escolar também pode contribuir positivamente para a educação sexual, visto que é um local onde o adolescente passa grande parte do seu tempo. Conforme é estipulado nos Parâmetros Curriculares Nacionais essa temática deve ser abordada de modo transversal em todas as disciplinas ministradas, tornando o professor um importante orientador do comportamento sexual seguro. No entanto, atualmente no Brasil, os documentos que norteiam as políticas públicas da educação veem silenciando de maneira expressiva os temas orientação sexual e sexualidade (LINS LS, et al., 2017).

Em pesquisa realizada por Souza Junior PR (2018) que teve como objetivo analisar as questões de gênero, sexualidade e orientação sexual na atual Base Nacional Comum Curricular (BNCC), observou-se que ocorreu um retrocesso sobre a discursão de gênero e sexualidade, no Plano Nacional de Educação (PNE) e na BNCC, documentos que suprimiram tal debate, atendendo aos pedidos da bancada fundamentalista / tradicional presente no Congresso Nacional.

Para Souza Júnior PR (2018), diante desta atual realidade, é necessário que cada vez mais seja aberto espaços para discussões e reflexões acerca deste retrocesso, principalmente em ambientes de construção de conhecimentos, afim de que possa ser fortalecido a inserção dos temas sexualidade e gênero no contexto escolar. Furlanetto MF, et al. (2018) também compreendem que houve um retrocesso nas políticas educacionais com a retirada da educação sexual na Base Nacional Comum Curricular e infere que precisamos avançar nas leis, estudos e ações de intervenções educativas dentro da escola. Mesmo com a implementação de algumas políticas neste setor, no Brasil, ainda é baixo o nível de informação dos adolescentes sobre sua sexualidade, fato que repercutirá diretamente nas suas atitudes, decisões e nos seus padrões comportamentais e sexuais, tornando-os vulneráveis a prejuízos no seu bem-estar biopsicossocial que podem ter efeitos negativos profundos em suas vidas (LINS LS, et al., 2017; MORAES SP e VITALLE MSS, 2015).

O desenvolvimento de uma educação sexual com informações adequadas, direciona o adolescente a reflexão crítica de sua realidade, favorecendo a vivência de atitudes e comportamentos saudáveis que promovem o senso de auto responsabilidade e compromisso para com a sua própria sexualidade (FAIAL LCM, et al., 2016; TORQUATO BGS, et al., 2017).

A ausência de um espaço adequado para discussão de um tema tão relevante para o desenvolvimento do adolescente como a sexualidade, gera sentimentos de ansiedade, culpa e medo, devido à falta de oportunidade de conhecer sua sexualidade como algo natural e sem preconceitos (TORQUATO BGS, et al., 2017). 
Diante deste contexto, objetivou-se com esta pesquisa identificar as principais dúvidas e medos com relação a sexualidade e analisar a percepção dos adolescentes no enfrentamento destes.

\section{MÉTODOS}

Trata-se de uma pesquisa descritiva e de natureza qualitativa, realizada com adolescentes escolares do 6 ao 9o ano do ensino fundamental da rede municipal de Belo Jardim - Pernambuco situado na região Nordeste e da rede Estadual de Uruguaiana - Rio Grande do Sul região sul do Brasil desenvolvida através de um questionário autoaplicável com perguntas abertas sobre dúvidas e medos acerca da sexualidade construído pelos autores com base na literatura. A escolha das escolas deu-se em virtude de estarem localizadas nos municípios que os autores residiam.

Dentre os critérios de inclusão adotados na pesquisa estavam: ser adolescente, ou seja, indivíduos com idade entre 12 a 15 anos e estar regulamente matriculado no ensino fundamental. Como critérios de exclusão elencou-se: ter faltado à aula no dia da coleta, não aceitar participar da pesquisa, participando do estudo, 38 adolescentes.

O instrumento para a coleta de dados foi construído pelos autores, composto pela seguinte questão aberta: "Com relação à sexualidade, destaque as principais dúvidas e medos". Antes da coleta, foi realizado teste piloto, visando testar o instrumento de pesquisa, avaliando a clareza das perguntas e a eficácia do instrumento.

As informações coletadas foram submetidas à Análise de Conteúdo. Para isso, foram realizadas as etapas de: pré-análise, com o objetivo de identificar estruturas relevantes através da leitura exaustiva; exploração do material que consiste em identificar no texto os núcleos de sentido e organizá-los; e o tratamento dos resultados, inferência e interpretação em que ao se descobrir o significado da regularidade, as informações foram analisadas, sendo possível realizar interpretações inferenciais, críticas e reflexões (BARDIN L, 2011).

Assim, a interpretação dos dados se deu pelo método de Análise de Conteúdo. Os dados foram importados para o programa de análise de dados qualitativos de licença livre $\mathrm{N}$-vivo, a fim de facilitar a análise qualitativa para a construção da nuvem de palavras, que foram formatadas a partir da análise de frequência com sinônimos, considerando as 1000 palavras mais frequentes.

Após a análise, os dados foram expostos através de gráfico de nuvens de palavras, bem como das respostas dos participantes, quando se fez importante para a compreensão do objeto de estudo, assegurando o sigilo e anonimato dos participantes, sendo estes classificados, para fins de organização dos dados de pesquisa, através de numeração arábica (Participante 1, Participante 2, etc.).

O estudo foi apreciado e aprovado pelo Comitê de Ética em Pesquisa da Autarquia Educacional do Belo jardim conforme a Resolução № 466/12, referente aos aspectos éticos da pesquisa envolvendo seres humanos, sob o número do Parecer: 828.470. Os dados foram coletados somente após explicação sobre os objetivos da pesquisa, sendo garantido de sigilo das informações por parte dos pesquisadores. Foram assinados o Termo de Compromisso Livre e Esclarecido (TCLE) pelos pais e o Termo de Assentimento pelos (as) adolescentes que aceitaram participar voluntariamente da pesquisa.

\section{RESULTADOS}

No que diz respeito ao perfil dos 38 adolescentes participantes da pesquisa, observou-se que a maioria era do sexo feminino, representando $52,7 \%$ (20) e $47,3 \%$ (18) eram do sexo masculino, com faixa etária média de 13 anos. Em relação a renda, a maioria $(41,67 \%)$ referiu ter entre 1 a 2 salários mínimos como renda da família. Ao serem questionados sobre suas principais dúvidas sobre a temática sexualidade, termos como "dúvidas", "doença" e "AIDS" foram frequentes, como se pode observar através da nuvem de palavras exposta na Figura 1. 
Figura 1 - Nuvem de palavras sobre "principais dúvidas relacionadas a sexualidade".

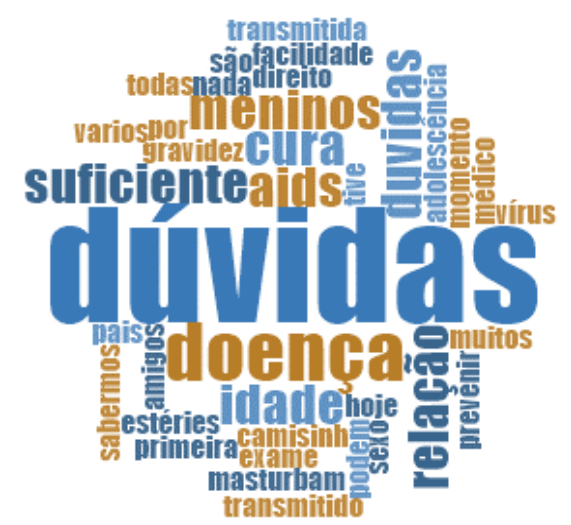

Fonte: Barbosa LU, et al., 2020.

Temas como "gravidez" relacionados à prática sexual foram citadas pelos adolescentes, como podemos exemplificar no trecho a seguir:

"Como acontece a gravidez, se fazer sexo com vários o que isso pode causar" (Participante 2).

Por outro lado, alguns participantes referiram não ter dúvidas relacionadas a sexualidade, indicando que a prática do diálogo com os pais e amigos auxiliam neste processo. Já a questão da idade relacionada ao conhecimento sobre sexualidade também foi citada pelo Participante 9, indicando uma possível relação entre o fornecimento das informações sobre a temática mediante a idade do adolescente, conforme observa-se na resposta a seguir:

"Dúvidas não tenho pois converso bastante com meus pais e amigos" (Participante 3).

"Nenhuma (bem pra minha idade eu já sei o suficiente, espero aprender um pouco a mais)" (Participante 9).

Outro questionamento realizado aos adolescentes durante a pesquisa foi pertinente aos principais medos dos mesmos em relação a sua sexualidade. Os termos "doença", "engravidar" e "medo" apareceram com grande frequência nas respostas dos participantes. Outros termos como "certo", "errado" e "filhos" foram citados pelos adolescentes. Os termos referidos pelos participantes em resposta a esse questionamento estão representados abaixo (Figura 2).

Figura 2 - Nuvem de palavras sobre "principais medos relacionados a sexualidade"

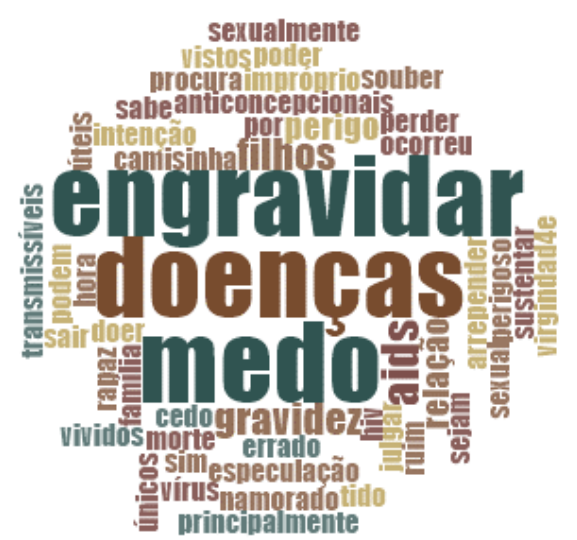

Fonte: Barbosa LU, et al., 2020. 
No tocante aos principais medos relatados pelos adolescentes, surgiram principalmente a gravidez indesejada e a contaminação por IST's. Além dessas temáticas, é possível observar que questões afetivas e sociais permeiam o universo da sexualidade vivenciada por estes. Os depoimentos que seguem vêm corroborar como este entendimento.

"Gravidez, doenças, que eu goste do rapaz, mas a intenção dele seja ruim" (Participante 2).

\author{
"Gravidez e doenças" (Participante 3). \\ "Tenho medo de engravidar antes da hora" (Participante 4). \\ "Tenho medo de engravidar cedo, de pegar algum tipo de doenças e \\ principalmente perder a virgindade e meu namorado me deixar e ainda espalhar \\ para todos o que ocorreu" (Participante 5).
}

\title{
DISCUSSÃO
}

Observou-se nos resultados que os adolescentes referiram dúvidas sobre a temática sexualidade, ressaltando aquelas relacionadas a doenças e gravidez. Neste sentido, apesar da vida sexual iniciar cada vez mais cedo nos últimos tempos, percebe-se que os adolescentes não têm acesso a informações consistentes que influenciem positivamente na prática sexual. A fonte destas informações é, na maioria das vezes, amigos e colegas, os quais também não tiveram acesso a uma educação sexual confiável (CAMILO VMB, et al., 2009; ALMEIDA RAAS, et al., 2017).

Estudos apontaram que dentre os fatores que contribuem para a gravidez na adolescência destaca-se más condições de educação e saúde, o que implica em dúvidas e práticas sexuais não seguras. Observa-se nas respostas dos adolescentes participantes dessa pesquisa o temor de uma gravidez precoce, assim como em outros estudos, esse sentimento se associa com a ausência de informações sobre 0 funcionamento do próprio corpo, métodos anticoncepcionais e o uso correto desses métodos (ALMEIDA RAAS, et al., 2017; MENDES MAR, et al., 2016).

Compreende-se que a gestação nessa fase da vida está associada a problemas como abandono escolar, falta de apoio financeiro, fuga da responsabilidade por parte do parceiro, rejeição familiar no início da gravidez, dentre outros provocando uma reformulação dos planos de vida dessas jovens (MENDES MAR, et al., 2016). Ainda em consequência da gravidez precoce, as adolescentes descreveram a existência de forte estresse, falta de apoio familiar e evasão escolar, culminando em implicações socioeconômicas que podem permanecer por toda a vida (TORRES JDRV, et al., 2018; RODRIGUES KA, et al., 2018).

Corroborando com esta pesquisa, um estudo com puérperas adolescentes identificou que o conhecimento sobre o ciclo menstrual e o período fértil, bem como no que se refere a métodos contraceptivos se mostrou limitado, a escola e os profissionais de saúde foram citados como as principais fontes de informação (LARA MO, et al., 2018). Pesquisas apontam que a ausência da escola como mais um espaço importante e necessário no diálogo e aprendizado acerca da sexualidade e saúde reprodutiva muitas vezes está relacionado com a falta de formação docente, logo os professores não se sentem preparados para abordar temas como IST's e saúde reprodutiva (CALDEIRA E e LOPES MJ, 2017).

Os dados encontrados nesta pesquisa convergem parcialmente com os do estudo realizado com adolescentes do Ensino de Jovens e Adultos (EJA) que salientou que a maioria dos participantes referiam conversar sobre a temática sexualidade com adolescentes do mesmo sexo ou sexo oposto e namorado (MACEDO SRH, et al., 2013). O diálogo no seio familiar sobre este tema foi minimamente citado, demonstrando um receio em realizá-lo, atribuído ao tom repressivo e inibidor que permeia este assunto, o que nos remete a atitude baseada na crença dos pais em que estimular o diálogo sobre sexualidade serve como indutor de uma prática sexual precoce, desconsiderando todos os outros fatores relacionados com a sexualidade como a gravidez e as IST's (MENDES MAR, et al., 2016; FREIRE AKS, et al., 2017). Este tabu pode estar relacionado ao fato de que a palavra sexualidade é compreendida de forma reduzida, apenas se 
restringindo ao ato sexual em si (RODRIGUES KA, et al., 2018; FREIRE AKS, et al., 2017). Logo, concordase com Nascimento BS, et al. (2017) ao inferirem que é urgente e necessário promover aos adolescentes espaço de diálogo e discussões acerca da sexualidade e as vulnerabilidades de maneira aberta e sem julgamentos, para que seja construído conhecimento que favoreça uma saúde sexual e reprodutiva segura e saudável.

Em geral, o medo está intimamente relacionado a falta de informação adequada sobre determinado assunto (FREIRE AKS, et al., 2017). Estudo realizado em uma escola pública de ensino fundamental e médio de Fortaleza - Ceará, identificou que os adolescentes têm pouco ou nenhum conhecimento sobre sintomas, transmissão e formas de prevenção das IST's (CAMILO VMB, et al., 2009), dado que corrobora com os resultados encontrados neste estudo, quando vimos através das respostas dos participantes que um dos principais medos relatados foi relacionado a estas doenças.

A relação familiar também é um fator que incide no desenvolvimento de práticas sexuais seguras e menor incidência de IST's. Em pesquisa realizada na cidade de Caicó - RN, identificou-se que as boas relações familiares influenciam o menor percentual de sujeitos com IST's, sendo suporte na vulnerabilidade relacionada às doenças (CORDEIRO JKR, et al., 2017).

Também foi possível observar que o conhecimento limitado sobre sexualidade e prevenção de IST's contribuiu para práticas sexuais de risco como o início da atividade sexual precocemente, a diminuição da frequência do uso de preservativos e a multiplicidade de parceiros, favorecendo assim o surgimento essas doenças. Desta forma pode-se relacionar as dúvidas e medos citados pelos participantes deste estudo a uma possível falta de conhecimento sobre a temática, que pode levar, consequentemente, a um comportamento sexual inseguro, tornando-as mais vulneráveis as situações de gravidez precoce e IST's/AIDS (RODRIGUES MGS, et al., 2010; CORDEIRO JKR, et al., 2017).

Assim como nos resultados desta pesquisa, o medo relacionado ao diagnóstico de uma IST foi referido por adolescentes. Os mesmos relacionaram a existência da IST com a dor física, o medo da morte e do preconceito que a sociedade tem com estas pessoas, também sendo referido receio do isolamento social. Apesar do investimento em campanhas que abordem a prevenção da transmissão de IST's, principalmente através do uso do preservativo masculino, nota-se que ainda existe uma lacuna em relação a efetividade destas informações, e o quanto a educação sexual nos moldes praticados contemporaneamente atingem de maneira eficaz a população adolescente (RODRIGUES MGS, et al., 2010; CORDEIRO JKR, et al., 2017).

Desta forma, infere-se que práticas educativas com estratégias eficazes para os adolescentes, envolvendo-os e empoderando-os de conhecimento e informações seguras podem contribuir positivamente para a minimização das dúvidas e, consequentemente, dos medos referidos frequentemente por estes. A presença de um profissional de educação junto com os profissionais de saúde inseridos no contexto escolar, para guiar este espaço de diálogo é essencial, pois a escola é o melhor espaço para realizar ações preventivas com adolescentes, promovendo uma vida saudável não apenas em termos de sua sexualidade, mas sensibilizando-os ao desenvolvimento da autoestima, para que saibam fazer escolhas, posicionando-se de forma autônoma frente a situações e responsabilizando-se por suas decisões (RODRIGUES MGS, et al., 2010).

Por fim, é nítido que o contexto escolar é de suma importância para melhor esclarecer e empoderar os adolescentes a respeito da sexualidade e todo o seu contexto. No entanto, a escola precisa estar preparada para apresentar reflexões que possam gerar pensamentos e opiniões críticas entre os (as) estudantes, motivando a reflexão sobre o acesso de todos à cidadania, respeitando e valorizando a diversidade. Bem como, não se pode deixar de levar em consideração que o contexto familiar também pode e deve contribuir na educação sexual dos adolescentes, visando, principalmente IST's e gravidez precoce.

\section{CONSIDERAÇÕES FINAIS}

A partir deste estudo foi possível observar que o medo em contrair uma IST e de uma gravidez precoce, bem como as dúvidas quanto as questões que envolvem a sexualidade, estão muito presentes entre os 
adolescentes. Logo, o estudo aponta a necessidade de uma prática mais efetiva da educação sexual, principalmente no contexto escolar com a participação dos adultos de referência (pais e/ou responsáveis, profissionais da educação e da saúde) de maneira transversal, que possa tornar a informação acessada pelo adolescente segura. Influenciando assim na sua perspectiva de mundo, fortalecendo o empoderamento e um comportamento sexual seguro, impactando diretamente na redução de indicadores de ISTs e gravidez precoce, que são situações que interferem na qualidade de vida dos adolescentes. Sugere-se que novos estudos sejam desenvolvidos nesta temática, para fortalecer o conhecimento científico e subsidiar o debate nos espaços relacionados a este diálogo.

\section{REFERÊNCIAS}

1. ALMEIDA RAAS, et al. Knowledge of adolescents regarding sexually transmitted infections and pregnancy. Rev Bras Enferm, 2017; 70(5): 1033-1039.

2. BARDIN L. Análise de conteúdo. Lisboa: Edições 70, 2011.

3. CALDEIRA E, LOPES MJ. Educação Sexual na Escola Contextos Para a Mudança. Rev. Ibero-Americana de saúde e envelhecimento, 2017; 3(3): 1147-1164.

4. CAMILO VMB, et al. Educação em saúde sobre DST/AIDS com adolescentes de uma escola pública, utilizando a tecnologia educacional como instrumento. DST - J Bras Doenças Sex Transm, 2009; 21(3): 124-128.

5. CAMPOS HM. O sujeito adolescente e o cuidado de si: cenários, significados e sentidos da iniciação sexual e do cuidado com a saúde sexual e reprodutiva. Dissertação (Mestrado em Ciências da Saúde) - Centro de Pesquisas René Rachou. Fundação Oswaldo Cruz, Belo Horizonte, 2011; 329p.

6. CORDEIRO JKR, et al. Adolescentes escolares acerca das DST/AIDS: quando o conhecimento não acompanha as práticas seguras. Rev enferm UFPE on line, 2017; 11(7): 2888-2896.

7. FAIAL LCM, et al. A escola como campo de promoção à saúde na adolescência: revisão literária. Revista PróUniver SUS, 2016; 7(2): 22-29.

8. FREIRE AKS, et al. Aspectos psicossociais da sexualidade na adolescência: diálogos e aprendizagem na escola. Semina: Ciências Biológicas e da Saúde, 2017; 38(1): 3-14.

9. FURLANETTO MF, et al. Educação sexual em escolas brasileiras: revisão sistemática da literatura. Cadernos de Pesquisa, 2018; 48(168): 550-571.

10. LARA MO, et al. Aspectos Sociodemográficos, História Sexual Reprodutiva e Conhecimento Contraceptivo de Puérperas Adolescentes e Adultas. RECOM, 2018; 8: 2845-2857.

11. LINS LS, et al. Análise do comportamento sexual de adolescentes. Rev Bras Promoç Saúde, 2017; 30(1): 47-56.

12. MACEDO EOS, CONCEIÇÃO MIG. Significações sobre Adolescência e Saúde entre Participantes de um Grupo Educativo de Adolescentes. Psicologia: ciência e profissão; 2015; 35(4): 1059-1073.

13. MACEDO SRH, et al. Adolescência e sexualidade: scripts sexuais a partir das representações sociais. Rev. bras. enferm., 2013; 66 (1): 103-109.

14. MENDES MAR, et al. Vamos falar sobre gravidez na adolescência? Experiência de educação e saúde em um quilombo. Revista UNIMONTES CIENTÍFICA, 2016; 18(2): 158-170.

15. MORAES SP, VITALLE MSS. Direitos sexuais e reprodutivos na adolescência: interações ONU-Brasil. Ciência \& Saúde Coletiva, 2015; 20(8): 2523-2531

16. NASCIMENTO BS, et al. Comportamento sexual de jovens universitários e o cuidado com a saúde sexual e reprodutiva. Enferm. glob., 2017;17(1): 237-69.

17. QUEIROZ VR, ALMEIDA JM. Sexualidade na adolescência: potencialidades e dificuldades dos professores de ensino médio de uma escola estadual de Sorocaba. Rev Fac Ciênc Méd Sorocaba, 2017; 19(4): 209-14.

18. RODRIGUES KA, et al. Gravidez e doenças sexualmente transmissíveis na adolescência. Arq. Catarin Med, 2018; 47(2): 212-225.

19. RODRIGUES MGS, et al. Talleres educativos en sexualidad del adolescente: la escuela como escenario. Enferm. glob., 2010; (20): 1-8.

20. SOUZA JUNIOR PR. A questão de gênero, sexualidade e orientação sexual na atual base nacional comum curricular (BNCC) e o movimento LGBTTQIS. Revista de Gênero, Sexualidade e Direito, 2018; 4(1): 1-21.

21. TORQUATO BGS, et al. O saber sexual na adolescência. Rev. Ciênc. Ext, 2017; 13(3): 54-63.

22. TORRES JDRV, et al. O significado da maternidade para adolescentes atendidas na Estratégia de Saúde da Família. Rev Fun Care Online, 2018; 10(4): 1003-1013. 